\title{
The Management of Endoscopic Retrograde Cholangiopancreatography-Related Duodenal Perforation
}

\author{
Kwang Bum Cho \\ Division of Gastroenterology and Hepatology, Department of Internal Medicine, Keimyung University School of Medicine, Daegu, Korea
}

\begin{abstract}
Uneventful duodenal perforation during endoscopic retrograde cholangiopancreatography (ERCP) is an uncommon but occasionally fatal complication. ERCP-related perforations may occur during sphincterotomy and improper manipulation of the equipment and scope. Traditionally, duodenal perforation has been treated with early surgical repair. Recently, nonoperative early endoscopic management techniques including clips or fibrin glue have been reported. In the present paper we review the literature pertaining to the treatment of perforations.
\end{abstract}

Key Words: Cholangiopancreatography, endoscopic retrograde; Perforation; Stents

\section{INTRODUCTION}

Endoscopic retrograde cholangiopancreatography (ERCP) is an invasive procedure carrying potential complications such as pancreatitis, hemorrhage, perforation, cholangitis, cholecystitis and cardiopulmonary abnormalities. ${ }^{1}$ Because of these complications, ERCP is used primarily for treatment rather than diagnostic purposes. The severity of ERCP-related complications varies from minimal to severe, which require surgical intervention or leave permanent sequelae. For those reasons, special requirements for endoscopists and the prolonged learning curve are necessary to avoid complications of ERCP, which occur in approximately $5 \%$ to $10 \%$ of patients..$^{2-4}$

Among the ERCP-related complications, perforations may occur during sphincterotomy, guidewire insertion or inadequate manipulation of the scope and equipment during bile duct stone removal. Moreover, duodenal wall perforations may occur as a result of the dislocation of the endoscopic retrograde biliary drainage. ${ }^{5,6}$ Although the incidence of perfora-

\section{Received: March 31, 2014 Revised: July 4, 2014}

Accepted: July 8, 2014

Correspondence: Kwang Bum Cho

Division of Gastroenterology and Hepatology, Department of Internal Medicine, Keimyung University School of Medicine, 56 Dalseong-ro, Jung-gu, Daegu 700-712, Korea

Tel: +82-53-250-7007, Fax: +82-53-250-7088, E-mail: chokb@dsmc.or.kr

(c) This is an Open Access article distributed under the terms of the Creative Commons Attribution Non-Commercial License (http://creativecommons.org/ licenses/by-nc/3.0) which permits unrestricted non-commercial use, distribution, and reproduction in any medium, provided the original work is properly cited. tion is lower than $1 \%$, the mortality rate from perforations is $\sim 16 \%$. Among the different types of perforation, perivaterian and bile duct perforations occur relatively often and respond well to medical treatments. Direct endoscopy-induced perforation of the duodenal wall is less common; however, the perforation tends to be large. ${ }^{3,7}$ Duodenal perforations result in considerable pancreatic and bile juice leakage, causing severe complications and a high mortality rate. Traditionally, the treatment for traumatic or iatrogenic duodenal perforation has been early surgical repair. Recently, the use of early endoscopy closure as a nonoperative management of duodenal wall perforation has increased ${ }^{8}$ however, the optimum treatment methods for duodenal perforation have not been established.

Therefore, in the present review we evaluated the literature pertaining to the prevention and treatment of ERCP-related perforations and the proposed methods for their management.

\section{THE CLASSIFICATION OF DUODENAL PERFORATIONS}

Stapfer et al. ${ }^{6}$ clinically classified perforations into four categories according to the severity (Fig. 1). ${ }^{9}$ Type I refers to perforation of the lateral or medial duodenal wall, which can cause leakage of a large volume of digestive juice and may occur as a result of tearing by the distal end of the endoscope or the migration of biliary stents. Type I perforations often cause 
large, persistent pancreatic juice leaks to the retroperitoneal or intraperitoneal space requiring immediate diagnosis and surgical repair. Type II refers to an ampullary perforation that occurs during sphincterotomy. The retroperitoneal space is the main site for accumulation of the leakage. Depending on the amount of digestive juice leakage and the size of perforation, type II perforations require either a surgical or medical treatment, such as stenting. ${ }^{10,11}$ Type III refers to perforations within the bile duct that occur during the insertion of a guidewire, treatment of the bile duct stone or in biliary strictures. In general, type III perforations are small and medical treatment with adequate drainage is sufficient. Type IV refers to retroperitoneal microperforations that can occur during sphincterotomy and result in air leakage to the retroperitoneal site. Type IV perforations are treated conservatively (Fig. 2). In response to the classification system established by Stapfer et al., ${ }^{6}$ Howard et al. ${ }^{5}$ classified perforations into the three following types: type I, perforation due to the guidewire; type II, periampullary perforation; and type III, perforation of the duodenal wall. Enns et al. ${ }^{12}$ also classified perforations into three types: type I, perforations of the esophagus, stomach, and duodenum, which require immediate surgery; type II, perforations associated with sphincterotomy; and type III, perforations caused by guidewires.

\section{CLINICAL CHARACTERISTICS}

The severity of perforations associated with ERCP can be

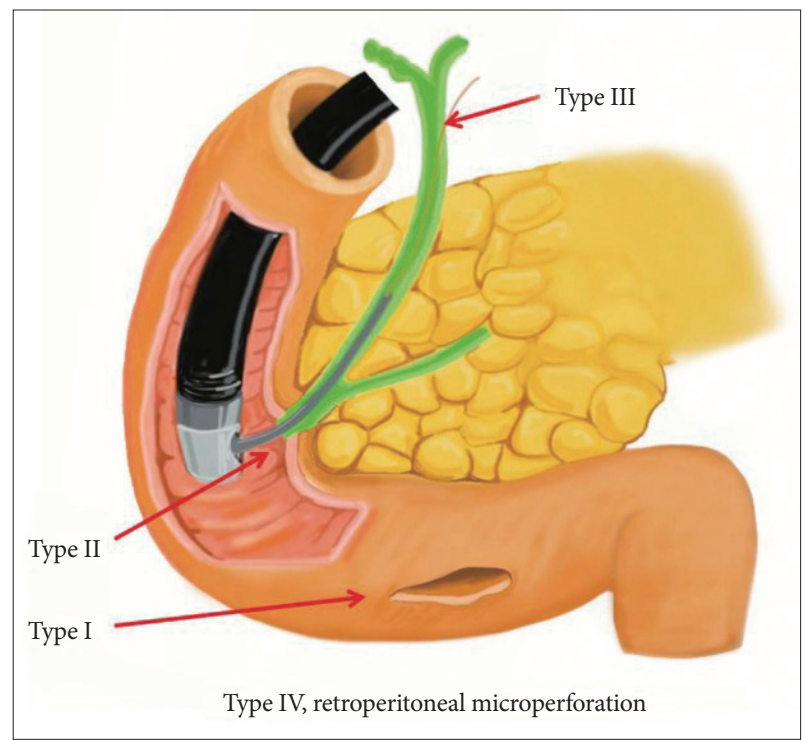

Fig. 1. Classification of iatrogenic duodenal perforations: type I, duodenal perforation due to endoscopy; type II, sphincterotomyrelated perforation; type III, biliary perforations due to endoscopic instruments such as guidewires; type IV, microperforations at the retroperitoneum during endoscopy. Adapted from Lee et al. Clin Endosc 2013;46:522-528. ${ }^{9}$ classified according to their symptoms. Minor perforations have minimal digestive juice leakage and require hospitalization of less than 3 days. A moderate degree of perforation requires 4 to 10 days of hospitalization with conservative treatment. A severe degree of perforation requires more than 10 days of conservative treatment, surgery or radiological intervention with percutaneous catheter drainage. ${ }^{1}$ Perforations may occur under various conditions, but the incidence is less than $1 \%{ }^{2-4,7,13,14}$ Risk factors for perforations include older age, sphincter of Oddi dysfunction, papillary stenosis, anatomical alteration due to surgery (e.g., Billroth II gastrectomy or Roux-en-Y gastric bypass), cases of precut method and prolonged ERCP duration. ${ }^{7,15}$ Symptoms of perforations range from an initial asymptomatic to severe abdominal pain, subcutaneous emphysema, peritonitis, abdominal distension, fever, cold sweating, and vomiting. ${ }^{10,16}$ However, even in cases of perforations, the initial stages are often asymptomatic or involve only minor symptoms. Furthermore, symptoms of perforation can easily be missed because the patient is under anesthesia and requires careful observation. Consequently, intraprocedural detection of contrast medium leakage or free air in the retroperitoneum and a postprocedural radiograph should be performed to confirm the presence of perforations. When the patient regains consciousness, a physical examination of the abdomen should be performed for the possibility of complications. Occasionally, the perforations may be accompanied by pancreatitis; in such cases, detection of perforations based on symptoms alone is difficult and so radiological examinations such as computed tomography (CT) should be performed to confirm their presence. Using CT scans, the

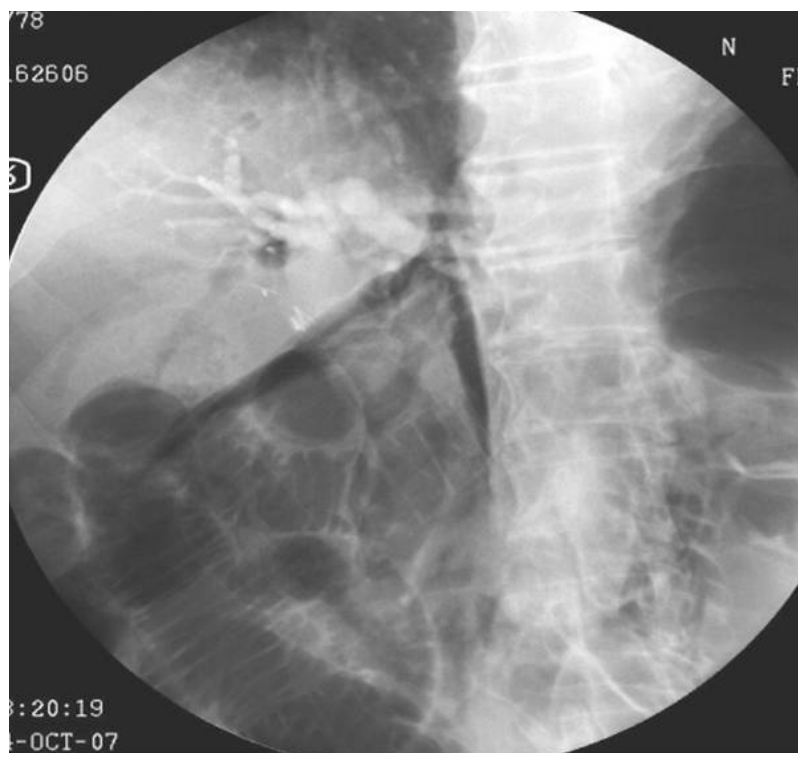

Fig. 2. Free air collection in the retroperitoneum after endoscopic sphincterotomy. 
presence of free air at the retroperitoneum may be detected in $29 \%$ of post-ERCP patients, including in asymptomatic subjects. ${ }^{17}$ Conversely, fluid collection in the retroperitoneum is more important for the prognosis of perforations. ${ }^{6,18}$

\section{SURGICAL TREATMENT}

Regarding perforation treatment, perforation sites, perforation type, degree of fluid leakage and symptoms determine the application of various treatment modalities. Surgery is performed in $20 \%$ to $50 \%$ of perforation patients, most of whom exhibit perforation of the duodenal or jejunal free wall. ${ }^{1,3,7,12,19,20}$ Free wall perforation generally causes large perforations and extensive leakage of the digestive fluid requiring immediate surgery upon diagnosis. Stapfer et al. ${ }^{6}$ recommended immediate surgical treatment in the following cases: large extravasations with incomplete dissipation 2 to 8 hours after ERCP, fluid collection in the retroperitoneum or peritoneum consistent with perforation and not pancreatitis based on CT images, documented ERCP perforation with common bile duct stones or retained hardware and detection of large amounts of subcutaneous emphysema with large duodenal diverticulum due to failure of conservative management.

Because the pathogenesis of perforations and radiological findings are well correlated, CT scans play an important role in determining the appropriate management modalities. The detection of free air in the retroperitoneum is not significantly associated with an adverse clinical prognosis. Consequently, if the CT scan shows only free air, conservative treatment should be performed. ${ }^{21}$ However, if the CT scan shows fluid accumulation, surgical treatment is recommended because fluid collection indicates continuous bile or pancreatic juice leakage through the perforation site. The choice of surgical repair is determined based on the patient's clinical condition and the perforation status.

\section{CONSERVATIVE TREATMENT}

Because periampullary perforations are relatively small and difficult to detect in the operating room, surgery is not recommended in most cases. If the bile and pancreatic juice leakage can be controlled, then conservative treatment is sufficient. Consequently, most periampullary perforations are treated conservatively, although serial radiographic examinations are necessary. In addition, even during conservative treatments, endoscopic nasobiliary or endoscopic nasopancreatic drainage should be performed to prevent continuous bile or pancreatic juice leakage through the perforation. However, if a large volume of leakage results in fluid collection in the retroperitoneum, then surgery or interventional drainage may be necessary. In nonsurgical perforation treatments, the cap-associated endoscopy method under direct vision through a transparent hood was helpful to ensure the safety margins during clipping (Fig. 3).9,22-24 The other nonsurgical treatment methods include an over-the-scope clip for ligation ${ }^{25}$ and spraying fibrin glue on the perforation site to block further fluid leakage. ${ }^{26,27}$ In addition, successful cases of self-expandable stent insertion in the periampullary perforation site to block additional bile or pancreatic juice leakage have been reported (Fig. 4). ${ }^{10,11}$

To avoid ampullary perforation during incision, the surgical knife wire should be inserted only slightly in the ampulla or bile duct to decrease the tension due to the knife. Then, the incision site should be observed carefully while making one small incision at a time. If a perforation is suspected during sphincterotomy, extravasations of contrast medium or free air
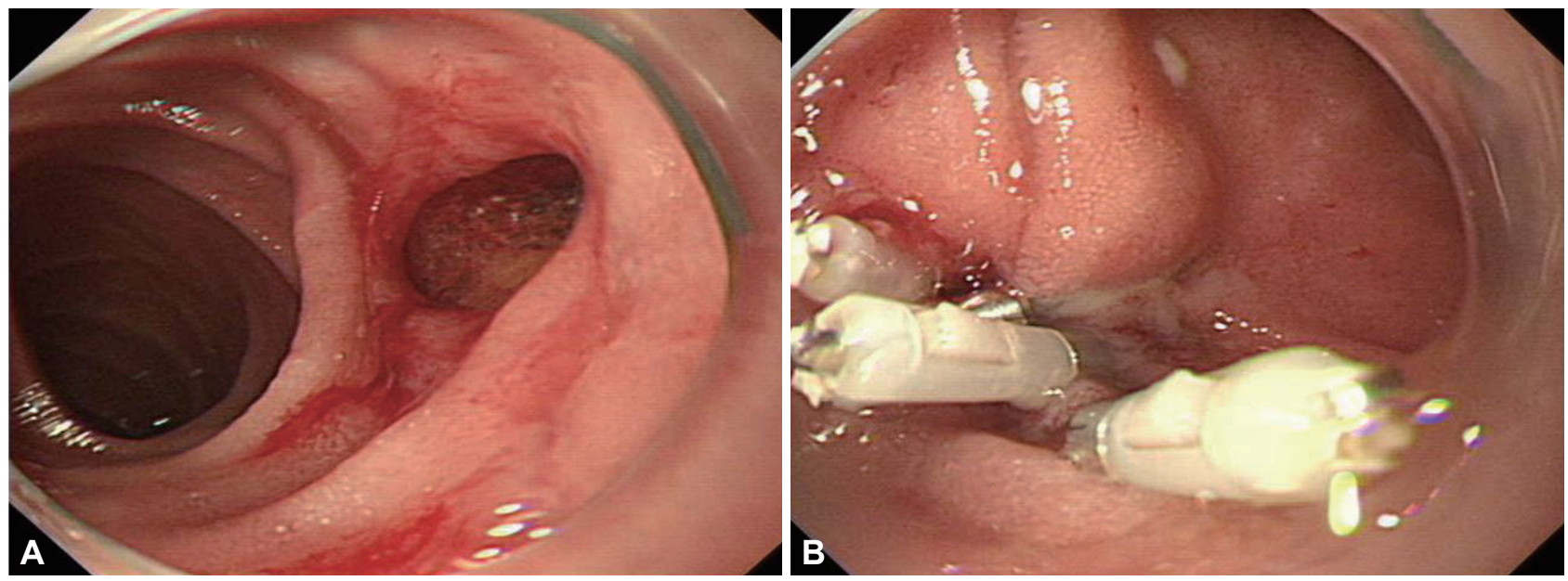

Fig. 3. Primary endoscopic closure of a duodenal wall perforation using endoclips. (A) Image obtained using a cap-assisted endoscope showing an ovoid perforation. (B) The perforation site was managed successfully using endoclips. 


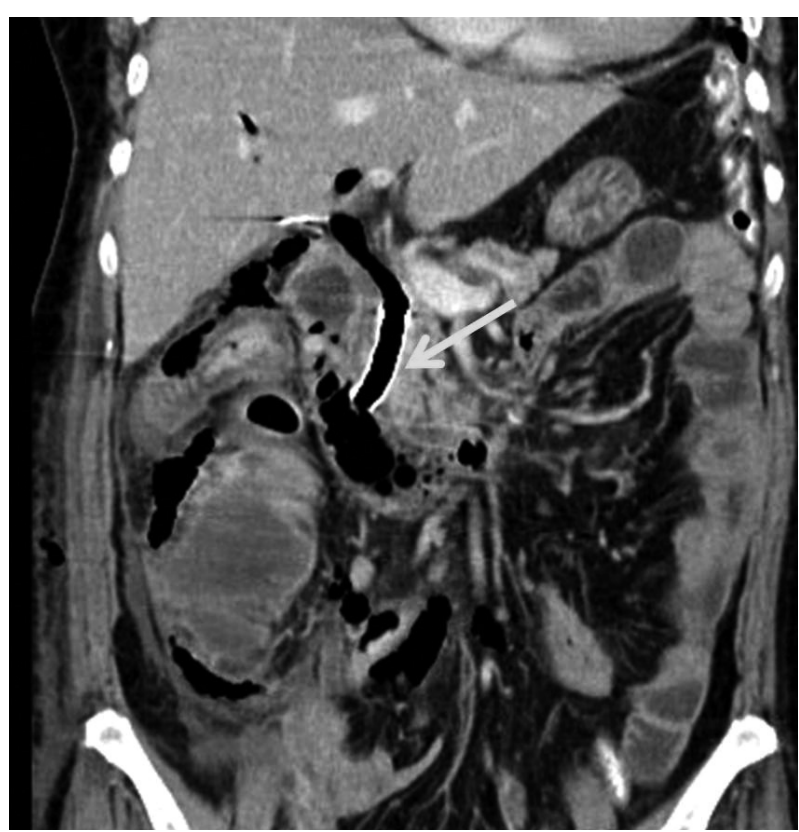

Fig. 4. Abdominal computed tomography scan showing fluid and air collection in the right retroperitoneum. To block further bile and pancreatic juice leakage, a self-expandable stent (arrow) was inserted at the distal common bile duct as conservative treatment.

in the retroperitoneal space should be inspected carefully. Additionally, biliary drainage or pancreatic duct drainage is recommended if possible before the end of the procedure. If perforation is still suspected, then an abdominal CT scan should be performed to determine the location and severity of the fluid or air leakage. If the leakage or the fluid accumulation increases or the patient's condition worsens despite the appropriate conservative management, although associated with a high risk, surgical treatment should be considered.

Because duodenal perforations are typically large and involve a large volume of bile leakage, surgical repair is usually required. However, an increased number of duodenal perforations treated successfully with endoclips or endoloops has been reported.

Generally, perforations within the bile duct caused by guidewires or stents are small and mostly asymptomatic; therefore, conservative treatment by appropriate endoscopic drainage is preferred. ${ }^{5,12}$

In several cases, plastic stent migration can cause perforations at the lateral duodenal wall. Because such perforations occur most commonly when using stents that are either linear in shape or of hard consistency in curvature, selection of the appropriate plastic stent according to the stricture location is necessary. If a duodenal perforation is caused by stent dislocation, one approach is to remove the stent and clipping followed by application of fibrin glue. ${ }^{27}$ However, in cases of peritonitis and fluid collection in the retroperitoneum, surgical repair is required. ${ }^{9}$
If conservative treatment is performed, biliary drainage is necessary to prevent further bile leakage from the perforation site. If endoscopic biliary drainage is difficult to perform, percutaneous transhepatic biliary drainage should be considered. Additionally, nasogastric drainage, fasting, intravenous proton pump inhibitor, antibiotic administration, surgical consultation, intensive treatment, and observation are required. Surgical treatment should be considered if consistent biliary obstruction or cholangitis occurs or the patient's condition worsens after conservative treatment. ${ }^{28}$

The prognosis of the perforation may depend on the time of detection and the overall condition. The mortality rate associated with perforations has decreased since 2000, likely due to early detection, advancement of conservative treatments, availability of treatment techniques, and the use of appropriate treatment methods.

\section{CONCLUSIONS}

Because ERCP can result in serious complications, the procedure should be substituted with either magnetic resonance cholangiopancreatography or endoscopic ultrasonography. Because the risk factors for various complications are relatively well-known, careful observation is necessary, especially in high-risk patients. The endoscopist's experience is closely associated with the incidence of complications; therefore, adequate experience and constant education are important. When a perforation occurs, the prognosis is influenced significantly by early diagnosis. Therefore, evaluation of intraprocedural changes in contrast medium or free air and the patient's postERCP condition by physical examinations or CT scans is important. Because the treatment of perforations depends on the characteristics of the perforation, the type of perforation should be determined as soon as possible. Moreover, as many treatment options-ranging from conservative to surgeryare available, advanced ligation tools and various types of endoclip should be readily accessible and endoscopists should be trained in their appropriate use. Additionally, consultation with surgeons as to the optimal treatment choice is important for perforation patients, and endoscopists should exercise caution to prevent potential complications.

\section{Conflicts of Interest}

The author has no financial conflicts of interest.

\section{REFERENCES}

1. Cotton PB, Lehman G, Vennes J, et al. Endoscopic sphincterotomy complications and their management: an attempt at consensus. Gastrointest Endosc 1991;37:383-393.

2. Freeman ML, DiSario JA, Nelson DB, et al. Risk factors for post-ERCP pancreatitis: a prospective, multicenter study. Gastrointest Endosc 
2001;54:425-434.

3. Loperfido S, Angelini G, Benedetti G, et al. Major early complications from diagnostic and therapeutic ERCP: a prospective multicenter study. Gastrointest Endosc 1998;48:1-10.

4. Williams EJ, Taylor S, Fairclough P, et al. Risk factors for complication following ERCP: results of a large-scale, prospective multicenter study. Endoscopy 2007;39:793-801.

5. Howard TJ, Tan T, Lehman GA, et al. Classification and management of perforations complicating endoscopic sphincterotomy. Surgery 1999; 126:658-663.

6. Stapfer M, Selby RR, Stain SC, et al. Management of duodenal perforation after endoscopic retrograde cholangiopancreatography and sphincterotomy. Ann Surg 2000;232:191-198.

7. Freeman ML, Nelson DB, Sherman S, et al. Complications of endoscopic biliary sphincterotomy. N Engl J Med 1996;335:909-918.

8. Nakagawa Y, Nagai T, Soma W, et al. Endoscopic closure of a large ERCP-related lateral duodenal perforation by using endoloops and endoclips. Gastrointest Endosc 2010;72:216-217.

9. Lee TH, Han JH, Park SH. Endoscopic treatments of endoscopic retrograde cholangiopancreatography-related duodenal perforations. Clin Endosc 2013;46:522-528.

10. Park WY, Cho KB, Kim ES, Park KS. A case of ampullary perforation treated with a temporally covered metal stent. Clin Endosc 2012;45:177180 .

11. Vezakis A, Fragulidis G, Nastos C, Yiallourou A, Polydorou A, Voros D. Closure of a persistent sphincterotomy-related duodenal perforation by placement of a covered self-expandable metallic biliary stent. World J Gastroenterol 2011;17:4539-4541.

12. Enns R, Eloubeidi MA, Mergener K, et al. ERCP-related perforations: risk factors and management. Endoscopy 2002;34:293-298.

13. Cheng CL, Sherman S, Watkins JL, et al. Risk factors for post-ERCP pancreatitis: a prospective multicenter study. Am J Gastroenterol 2006; 101:139-147.

14. Wang P, Li ZS, Liu F, et al. Risk factors for ERCP-related complications: a prospective multicenter study. Am J Gastroenterol 2009;104:31-40.

15. Wu HM, Dixon E, May GR, Sutherland FR. Management of perforation after endoscopic retrograde cholangiopancreatography (ERCP): a population-based review. HPB (Oxford) 2006;8:393-399.

16. Li G, Chen Y, Zhou X, Lv N. Early management experience of perfora- tion after ERCP. Gastroenterol Res Pract 2012;2012:657418.

17. Genzlinger JL, McPhee MS, Fisher JK, Jacob KM, Helzberg JH. Significance of retroperitoneal air after endoscopic retrograde cholangiopancreatography with sphincterotomy. Am J Gastroenterol 1999;94:12671270.

18. Zissin R, Shapiro-Feinberg M, Oscadchy A, Pomeranz I, Leichtmann G, Novis B. Retroperitoneal perforation during endoscopic sphincterotomy: imaging findings. Abdom Imaging 2000;25:279-282.

19. Fatima J, Baron TH, Topazian MD, et al. Pancreaticobiliary and duodenal perforations after periampullary endoscopic procedures: diagnosis and management. Arch Surg 2007;142:448-454.

20. Masci E, Toti G, Mariani A, et al. Complications of diagnostic and therapeutic ERCP: a prospective multicenter study. Am J Gastroenterol 2001;96:417-423.

21. Mao Z, Zhu Q, Wu W, et al. Duodenal perforations after endoscopic retrograde cholangiopancreatography: experience and management. J Laparoendosc Adv Surg Tech A 2008;18:691-695.

22. Baron TH, Gostout CJ, Herman L. Hemoclip repair of a sphincterotomy-induced duodenal perforation. Gastrointest Endosc 2000;52:566568.

23. Amodio PM, Faggiani R, Pastorelli A, et al. Selected treatments for duodenal perforation after ERCP. A report of three cases. Chir Ital 2007; 59:343-346.

24. Lee TH, Bang BW, Jeong JI, et al. Primary endoscopic approximation suture under cap-assisted endoscopy of an ERCP-induced duodenal perforation. World J Gastroenterol 2010;16:2305-2310.

25. Buffoli F, Grassia R, Iiritano E, Bianchi G, Dizioli P, Staiano T. Endoscopic "retroperitoneal fatpexy" of a large ERCP-related jejunal perforation by using a new over-the-scope clip device in Billroth II anatomy (with video). Gastrointest Endosc 2012;75:1115-1117.

26. Mutignani M, Iacopini F, Dokas S, et al. Successful endoscopic closure of a lateral duodenal perforation at ERCP with fibrin glue. Gastrointest Endosc 2006;63:725-727.

27. Kim KW, Lee TH, Park SH, et al. A primary repair with hemoclips and fibrin glue injection in biliary stent induced duodenal perforation. Korean J Gastrointest Endosc 2011;42:437-441.

28. Chung RS, Sivak MV, Ferguson DR. Surgical decisions in the management of duodenal perforation complicating endoscopic sphincterotomy. Am J Surg 1993;165:700-703. 